\title{
Dynamic susceptibility contrast and dynamic contrast-enhanced MRI characteristics to distinguish microcystic meningiomas from traditional Grade I meningiomas and high-grade gliomas
}

\author{
Namath S. Hussain, MD, MBA, ${ }^{1}$ Marc D. Moisi, MD, ${ }^{1}$ Bart Keogh, MD, PhD, ${ }^{1,2}$ \\ Brendan J. McCullough, MD, PhD, ${ }^{1,2}$ Steven Rostad, MD, ${ }^{1,3}$ David Newell, MD, ${ }^{1}$ Ryder Gwinn, MD,1 \\ Gregory Foltz, MD, ${ }^{1}$ Marc Mayberg, MD, ${ }^{1}$ Brian Aguedan, RT, ${ }^{2}$ Valerie Good, RT, ${ }^{2}$ and \\ Sarah J. Fouke, MD'
}

'Swedish Neuroscience Institute, Swedish Medical Center, and ${ }^{3}$ CellNetix Pathology and Laboratories, Seattle; and ${ }^{2}$ Radia Inc. PS, Everett, Washington

OBJECTIVE Microcystic meningioma (MM) is a meningioma variant with a multicystic appearance that may mimic intrinsic primary brain tumors and other nonmeningiomatous tumor types. Dynamic susceptibility contrast (DSC) and dynamic contrast-enhanced (DCE) MRI techniques provide imaging parameters that can differentiate these tumors according to hemodynamic and permeability characteristics with the potential to aid in preoperative identification of tumor type.

METHODS The medical data of 18 patients with a histopathological diagnosis of MM were identified through a retrospective review of procedures performed between 2008 and 2012; DSC imaging data were available for 12 patients and DCE imaging data for 6 . A subcohort of 12 patients with Grade I meningiomas (i.e., of meningoepithelial subtype) and 54 patients with Grade IV primary gliomas (i.e., astrocytomas) was also included, and all preoperative imaging sequences were analyzed. Clinical variables including patient sex, age, and surgical blood loss were also included in the analysis. Images were acquired at both 1.5 and 3.0 T. The DSC images were acquired at a temporal resolution of either 1500 msec (3.0 T) or $2000 \mathrm{msec}(1.5 \mathrm{~T})$. In all cases, parameters including normalized cerebral blood volume (CBV) and transfer coefficient (kTrans) were calculated with region-of-interest analysis of enhancing tumor volume. The normalized CBV and kTrans data from the patient groups were analyzed with 1-way ANOVA, and post hoc statistical comparisons among groups were conducted with the Bonferroni adjustment.

RESULTS Preoperative DSC imaging indicated mean ( \pm SD) normalized CBVs of $5.7 \pm 2.2 \mathrm{ml}$ for WHO Grade I meningiomas of the meningoepithelial subtype $(n=12), 4.8 \pm 1.8 \mathrm{ml}$ for Grade IV astrocytomas $(n=54)$, and $12.3 \pm 3.8 \mathrm{ml}$ for Grade I meningiomas of the MM subtype $(n=12)$. The normalized CBV measured within the enhancing portion of the tumor was significantly higher in the MM subtype than in typical meningiomas and Grade IV astrocytomas $(p<0.001$ for both). Preoperative DCE imaging indicated mean kTrans values of $0.49 \pm 0.20 \mathrm{~min}^{-1}$ in Grade I meningiomas of the meningoepithelial subtype ( $n=12), 0.27 \pm 0.12 \mathrm{~min}^{-1}$ for Grade IV astrocytomas ( $\left.n=54\right)$, and $1.35 \pm 0.74 \mathrm{~min}^{-1}$ for Grade I meningiomas of the MM subtype $(n=6)$. The kTrans was significantly higher in the MM variants than in the corresponding nonmicrocystic Grade 1 meningiomas and Grade IV astrocytomas ( $p<0.001$ for both). Intraoperative blood loss tended to increase with increased normalized CBV $(R=0.45, p=0.085)$.

CONCLUSIONS An enhancing cystic lesion with a normalized CBV greater than $10.3 \mathrm{ml}$ or a kTrans greater than 0.88 $\mathrm{min}^{-1}$ should prompt radiologists and surgeons to consider the diagnosis of MM rather than traditional Grade I meningioma or high-grade glioma in planning surgical care. Higher normalized CBVs tend to be associated with increased intraoperative blood loss.

https://thejns.org/doi/abs/10.3171/2016.3.JNS14243

KEY WORDS dynamic contrast-enhanced MRI; dynamic susceptibility contrast MRI; microcystic meningioma; astrocytoma; glioma; glioblastoma; oncology

ABBREVIATIONS CBV = cerebral blood volume; $\mathrm{DCE}=$ dynamic contrast-enhanced; $\mathrm{DSC}=$ dynamic susceptibility contrast; $\mathrm{kTrans}=$ transfer coefficient; $\mathrm{MM}=$ microcystic meningioma; NPV = negative predictive value; $\mathrm{PPV}=$ positive predictive value; $\mathrm{ROI}$ = region of interest.

SUBMITTED February 10, 2014. ACCEPTED March 22, 2016.

INCLUDE WHEN CITING Published online June 10, 2016; DOI: 10.3171/2016.3.JNS14243. 
$\mathrm{T}$ Raditionally, a radiographic diagnosis discriminating between intraaxial and extraaxial primary brain tumors is performed on anatomical MRI sequences. WHO Grade I meningiomas have a relatively characteristic, distinguishable appearance on MRI sequences, including T2-weighted and T1-weighted sequences with a contrast agent. A CSF cleft visible on T2-weighted sequences or a dural tail apparent on T1-weighted sequences with contrast agent are characteristics that are typically pathognomonic of these tumor types. ${ }^{3,4,16}$ Although advanced imaging sequences have been considered in the evaluation of typical meningiomas, ${ }^{19}$ physiological imaging sequences rarely affect the surgical management of these tumors.

Microcystic meningioma (MM) is an unusual variant of meningioma with a multicystic appearance that may mimic intrinsic primary brain tumors and other nonmeningiomatous tumor types. Dynamic susceptibility contrast (DSC) and dynamic contrast-enhanced (DCE) imaging modalities help differentiate certain tumor types according to physiological characteristics and have the potential to assist in the preoperative identification of this tumor type.

The MM variant often shows a radiographic appearance on anatomical sequences that can be challenging to identify as a meningioma and even to distinguish it as an extraaxial tumor. These rarer tumors have been described in the literature with an anatomical imaging appearance that may mimic intrinsic primary brain tumors, hemangioblastomas, and other nonmeningiomatous tumor types. ${ }^{3}$ Histopathologically, MM can cause diagnostic difficulty due to its peculiar nonmeningothelial pattern. The tumor can have intercellular, pale, and mucin-containing microcysts along with vacuolated, xanthomatous cells interspersed among hyalinized blood vessels. ${ }^{18}$ Other typical features such as lobules, whorls, and psammoma bodies are absent. $3,5,11$

As anatomical imaging sequences have not, in isolation, proven useful in distinguishing the MM variant from other tumor types, we have investigated DSC and DCE imaging techniques in the preoperative identification of MM. Dynamic susceptibility contrast imaging is also often referred to as "perfusion imaging" and has been widely used in the care of patients with stroke..$^{10,17,20}$ In essence, DSC involves the rapid acquisition of sequential images at a given location as a gadolinium $(\mathrm{Gd})$ contrast bolus moves through the brain vasculature, often termed the "first pass." From the first-pass curve, a quantitative measure of several physiological parameters may be derived, including normalized cerebral blood volume (CBV), cerebral blood flow, and mean transit time. ${ }^{6,9}$

The normalized CBV has been shown to correlate with astrocytoma grade $^{12}$ and is considered in the diagnostic distinction of tumor progression from radiation necrosis in primary brain tumors. ${ }^{15}$ Whereas the DSC technique is based on T2-weighted imaging sequences to indirectly measure capillary density and size, DCE imaging measures the rate of the T1 signal change after contrast agent administration to estimate capillary permeability and related parameters. The normalized CBV may also be measured with T1-weighted DCE techniques. ${ }^{12}$

As with the DSC technique, a number of metrics may be derived from DCE sequences, notably extracellular volume, measured with the extravascular, extracellular volume fraction, and permeability, measured with the transfer coefficient (kTrans). The kTrans, a volume-transfer constant of the contrast agent between blood plasma and the extravascular extracellular space, helps to determine vascular permeability and to evaluate the microvasculature within tumors. Both kTrans and the extravascular, extracellular volume fraction are good predictors of tumor grade, ${ }^{1,21}$ with correct classification of $92 \%$ of tumors in discriminant analysis. Therefore, our current standard of practice is to perform both sets of image acquisition.

This study aimed to use DSC and DCE characteristics on MRI sequences to distinguish MM from both traditional Grade I meningiomas and high-grade gliomas.

\section{Methods \\ Patient Cohort}

The medical records of 18 patients who had a histopathological diagnosis of MM between 2008 and 2012 were identified through a retrospective review performed at the Swedish Neuroscience Institute. Approval for review of the imaging sequences acquired as a part of the routine preoperative clinical care for these patients was obtained from the internal review board at Swedish Hospital before clinical research was initiated. A subcohort of 12 patients with Grade I meningiomas and of 54 with Grade IV primary gliomas was also included. This comparison group was a consecutive cohort seen by the neurosurgery service, and the preoperative imaging sequences of the patients in this group were also reviewed. The selection of the patient group sizes was based on a power analysis with an $80 \%$ probability of detecting a $20 \%$ difference between groups with an alpha value of 0.05 . Clinical variables including patient sex, age, and surgical blood loss were also analyzed. Blood loss during surgery was estimated from the surgeon's operative notes. None of the tumors in this study had been treated with preoperative embolization.

\section{Image Acquisition, Processing, and Analysis}

As a part of the clinical routine at our institution, a standard "tumor-protocol" MRI sequence is obtained in patients who present for resection. This imaging protocol includes 3D MP-RAGE/3D FSPGR noncontrast and Gd-enhanced volumetric acquisitions, T2-weighted and FLAIR sequences, susceptibility-weighted sequences, and diffusion-weighted imaging, acquired with diffusion tensor imaging ( 25 directions, b max $1000 \mathrm{msec} / \mathrm{mm}^{2}$ ). Since 2008 , with increasing frequency (and now currently routine), DSC imaging has been included in this tumor protocol to generate parameter maps of relative cerebral blood flow and normalized CBV. In addition, our imaging protocols have recently included DCE imaging before DSC imaging. Review of the data from the 18 patients with MM indicated that DSC imaging data were available for 12 of these patients and DCE imaging data for 6 (all of these 6 patients also underwent DSC imaging). The preoperative DSC and DCE imaging sequences of the 12 patients with Grade I meningioma (i.e., the meningoepithelial subtype) and 54 with Grade IV primary glioma were also reviewed. 
Images were acquired at both 1.5 and $3.0 \mathrm{~T}$ with $\mathrm{GE}$ instruments (LX, HDx, and MR750). The DSC images were acquired with a standard echo-planar imaging sequence at a temporal resolution of either $1500 \mathrm{msec}(3.0 \mathrm{~T})$ or $2000 \mathrm{msec}(1.5 \mathrm{~T})$, TE $25 \mathrm{msec}$, slice thickness $5 \mathrm{~mm}$, slice spacing $1 \mathrm{~mm}$, field of view $24 \mathrm{~cm}$, and matrix $128 \times$ 128. The DCE images were acquired with a standard 3D SPGR at a temporal resolution of $4 \mathrm{msec}$, TE $1.2 \mathrm{msec}$, slice thickness $5 \mathrm{~mm}$, slice spacing $0 \mathrm{~mm}$, flip angle $24^{\circ}$, and matrix $256 \times 160$

A Gd-based contrast agent (Gadovist, Bayer) was administered in 2 one-half boluses at a standard dose of 0.1 $\mathrm{mmol} / \mathrm{kg}$ with each the DCE (first) and DSC (second) sequences. By performing the DCE and DSC in sequence, preloading of the $\mathrm{Gd}$ contrast agent was accomplished. No mathematical leakage correction was used.

Image registration, postprocessing, and analysis were performed by experienced technical personnel certified by the American Registry of Radiologic Technologists and under supervision of a neuroradiologist (B.K.). The NordicICE software platform (Nordic NeuroLab), an FDA-approved, vendor-independent method for advanced imaging analysis, was used in these analyses. Analysis of regions of interest (ROIs) was performed with our standard clinical protocol, including exclusion of vessels and areas of qualitative necrosis. The ROIs were manually selected, focusing on enhancing tumor tissue.

\section{Histopathological Analysis}

Meningiomas with microcystic features were identified through a search of records of pathological diagnoses conducted over a 6-year period. Two microcystic patterns were observed in tumors of patients who had a diagnosis that included the term "microcystic meningioma." Pure MM tumors essentially comprised all conventional microcystic features, and in mixed MM tumors, the microcystic pattern comingled with other patterns. For this study, a neuropathologist re-reviewed the histopathological results for all of the included tumors to diagnose them as typical meningioma, high-grade primary brain tumor, MM, or mixed meningioma with microcystic features.

Histopathologically, MM may be difficult to accurately diagnose because of its peculiar nonmeningothelial pattern. The WHO classification was used as the primary method of tumor grading. ${ }^{2}$ The microscopic features most commonly associated with microcystic variants included hypervascularity, vascular hyalinization, micro- or macrocystic architecture with fluid-filled spaces, scattered nuclear pleomorphism, and xanthomatous changes (Fig. 1). $\mathrm{MM}$ is distinguished as having intercellular, pale, mucin-containing microcysts along with vacuolated, xanthomatous cells interspersed among hyalinized blood vessels. Other typical features such as lobules, whorls, and psammoma bodies are lacking, and ultrastructural studies can confirm the presence of desmosomes. ${ }^{3}$

For microcystic tumors defined as having a mixed pattern, at least $10 \%$ displayed changes observed in the pure MM subtype; however, other well-described patterns such as transitional or meningotheliomatous variants were also noted. In some cases, immunostaining was performed with appropriate positive and negative controls to detect

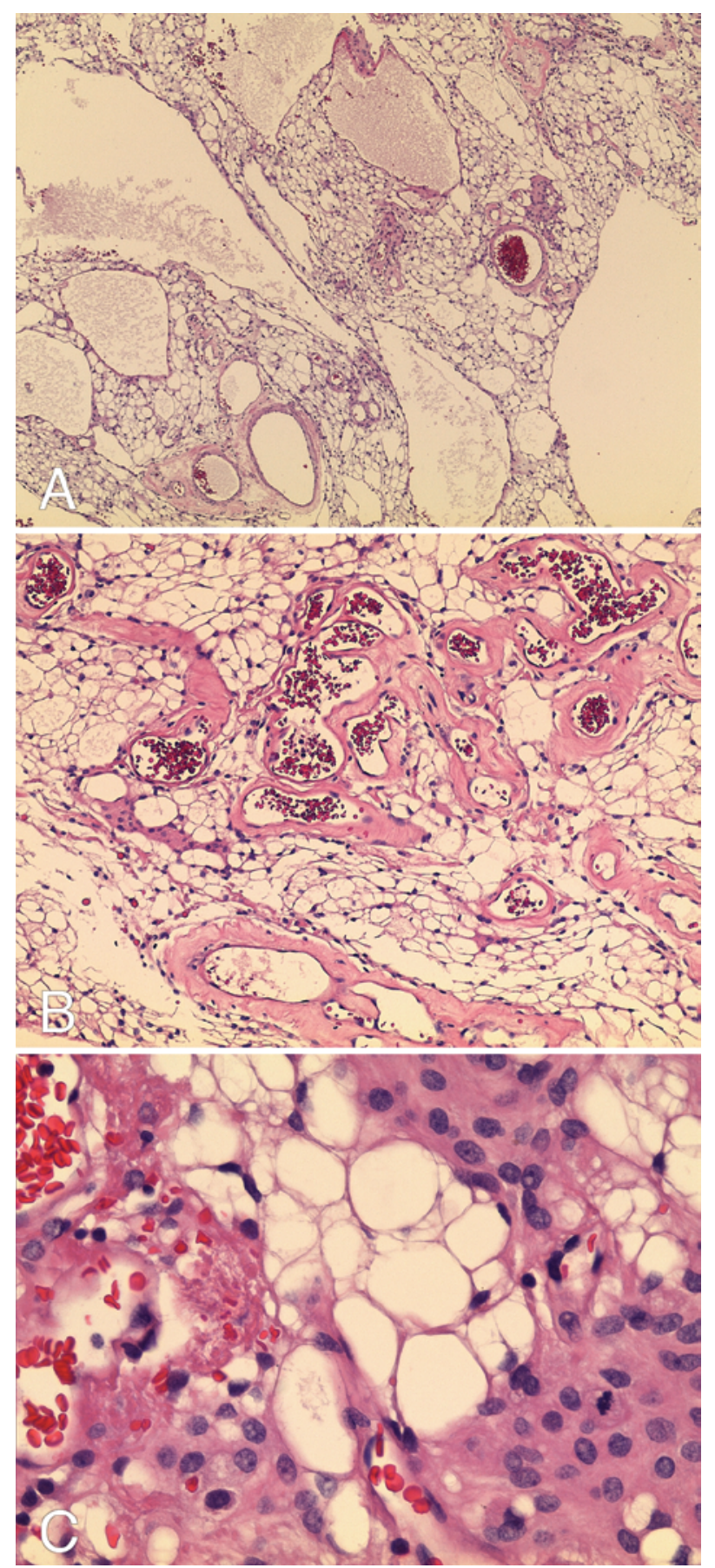

FIG. 1. Histopathological features of MM (with some but not all microcystic features) revealed by light microscopy of $\mathrm{H} \& \mathrm{E}$-stained tissue. A: Abundant spongy or fluid-filled cystic spaces of the MM, accounting for the soft and glistening cut surfaces of MMs. The cystic spaces range from macroscopic (i.e., grossly visible) to microscopic extracellular spaces. Increased vascularity of the tumor is present. Original magnification $\times 40$. B: Hypervascularity is a characteristic feature of MM; the vessels here show mild sclerosis. Original magnification $\times 100$. C: The delicate cytoplasmic processes separate the cystic spaces, and several smaller islands of solid meningotheliomatous differentiation are noted. Nuclear enlargement, hyperchromasia, and pleomorphism are common in the microcystic areas (not pictured here), but are not a marker for atypical or anaplastic meningioma. A rare mitotic figure is noted, although this example is a Grade I tumor. Original magnification $\times 400$. Figure is available in color online only. 
Ki-67 protein, epithelial membrane antigen, or progesterone receptor. Invasion of the cerebral cortex was occasionally evaluated with an immunostaining method for detecting glial fibrillary acidic protein.

\section{Statistical Analysis}

Imaging data were analyzed for all patients with nonmicrocystic meningioma and with MM and for those with intrinsic primary brain tumors. We statistically analyzed normalized CBV values with 1-way ANOVA, and post hoc comparisons between individual groups were performed with the Bonferroni method to adjust for multiple comparisons. Similar methods were used to evaluate differences in kTrans among the groups. The statistical analyses were performed with Stata 12 statistical software (StataCorp). Two-sided $p$ values were assessed at a significance level of alpha $=0.05$.

\section{Results}

Imaging data were analyzed for a total of 84 patients, including 12 with nonmicrocystic meningioma, 18 with $\mathrm{MM}$, and 54 with Grade IV primary glioma. Among the patients with MM (mean age 62.9 years, 7 men and 11 women), 9 had MMs with pure MM features, and 3 had tumors with a mixed histopathological appearance. Analysis of preoperative sequences with DSC imaging indicated enhancing tumor mean $( \pm \mathrm{SD})$ normalized CBV values of $5.7 \pm 2.2 \mathrm{ml}$ for Grade I meningiomas of the meningoepithelial subtype, $4.8 \pm 1.8 \mathrm{ml}$ for Grade IV astrocytomas, and $12.3 \pm 3.8 \mathrm{ml}$ for Grade I meningiomas of the MM subtype (Fig. 2). The normalized CBV measured within the enhancing portion of the tumor was significantly higher in the MM subtype than in both nonmicrocystic meningiomas and Grade IV astrocytomas ( $p<0.001$ for both). A representative $\mathrm{MM}$ with an elevated normalized CBV is shown in Fig. 3.

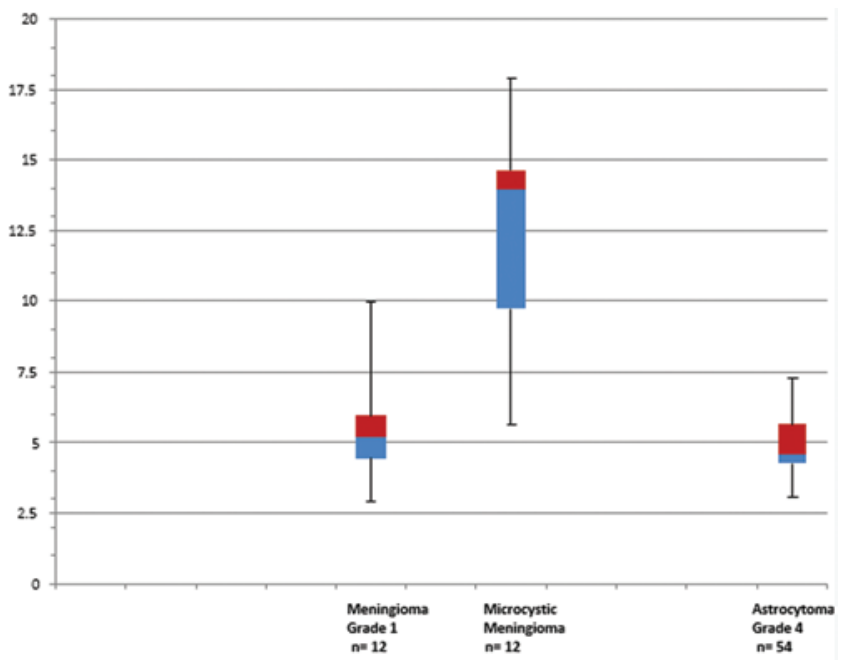

FIG. 2. Descriptive statistics of normalized CBV in patients with nonmicrocystic meningioma, MM, and Grade IV glioblastoma. Boxes represent the 25th-75th percentiles, the horizontal bars indicate the mean, and whiskers represent the data range. $n=$ number of patients. Figure is available in color online only.

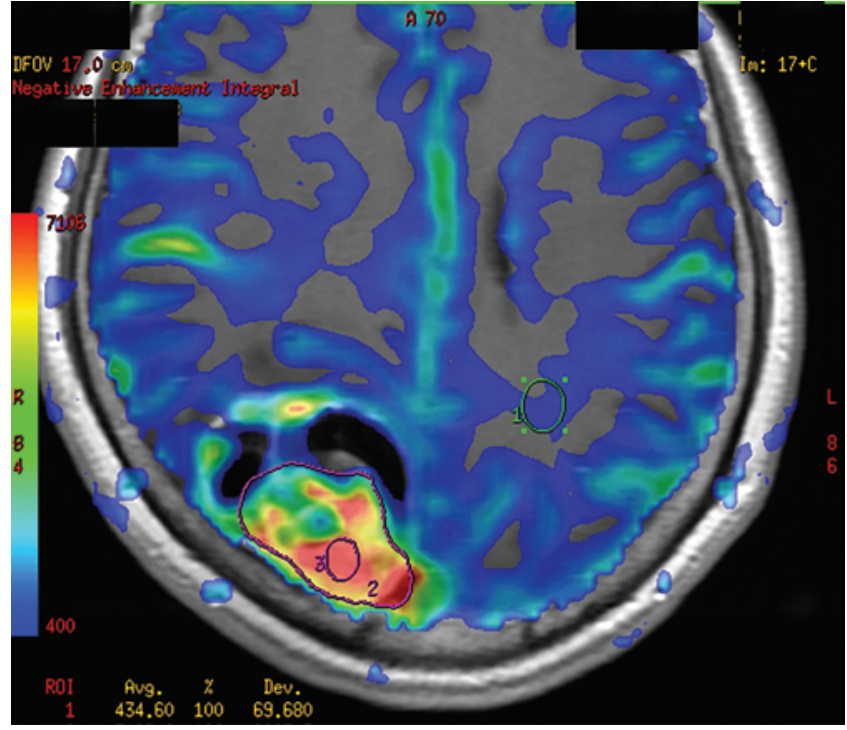

FIG. 3. Anatomical imaging sequences suggest an intraaxial mass, mostly likely high-grade glioma. Preoperative DSC imaging indicated a normalized CBV of $18 \mathrm{ml}$ within the enhancing tumor volume, a CBV that was higher than that typically observed in high-grade glioma. $\mathrm{A}$ measurement of relative CBV with the T2 and FLAIR abnormalities in the surrounding tissues indicated a normalized CBV of $<1.0 \mathrm{ml}$ in these tissues, again suggesting an extrinsic rather than intrinsic tumor. Histopathological analysis revealed MM. Figure is available in color online only.

Preoperative DCE imaging indicated $\mathrm{kTrans}$ values of $0.49 \pm 0.20 \mathrm{~min}^{-1}$ in Grade I meningiomas, $0.27 \pm 0.12$ $\mathrm{min}^{-1}$ in Grade IV astrocytomas, and $1.35 \pm 0.74 \mathrm{~min}^{-1}$ in MM. The kTrans measured within the enhancing portion of the tumor was significantly higher in the MM variants (Fig. 4) than in corresponding nonmicrocystic meningiomas (Fig. 5) and Grade IV astrocytomas ( $p<0.001$ for both) (Fig. 6). Primarily for simplicity and to broaden clinical usefulness, we decided a priori to compare normalized CBV and kTrans for the tumor types separately. A multivariate model including both measurements may provide a slightly higher likelihood of a correct preoperative diagnosis, but the added complexity would preclude clinical usefulness. We chose normalized CBV as the primary statistic because it is more widely available. In our sample, normalized CBV data were available for 12 patients, and kTrans data were available for one-half of the patients (i.e., 38 or $49 \%$ ), including only 6 patients with $\mathrm{MM}$ and 4 with meningoepithelial meningioma.

The data suggested that when the normalized CBV is greater than $10.3 \mathrm{ml}$ (i.e., the meningoepithelial mean plus 2 SDs) or the kTrans is above $0.88 \mathrm{~min}^{-1}$ (the meningoepithelial mean plus 2 SDs) in a patient with an enhancing tumor, a diagnosis of MM should be considered. Values for these 2 variables in Grade IV astrocytomas were both lower than for Grade I meningoepithelial tumors (the mean plus $2 \mathrm{SDs}$ was $7.7 \mathrm{ml}$ and $0.51 \mathrm{~min}^{-1}$ for normalized CBV and kTrans, respectively).

When we compared MM with Grade IV astrocytomas, we observed that a cutoff normalized CBV value of 10.3 $\mathrm{ml}$ yielded a positive predictive value (PPV) of $88 \%$ and a negative predictive value (NPV) of $91 \%$, with a specificity of $98 \%$ and sensitivity of $58 \%$ for comparing these 

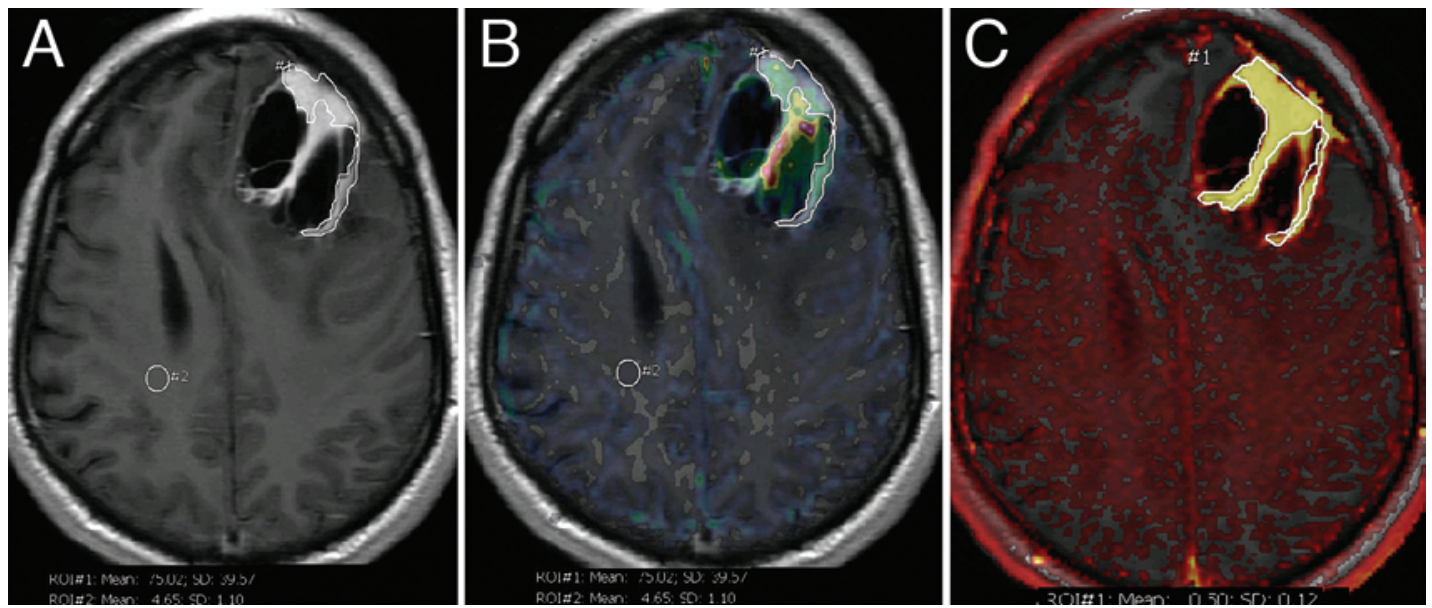

FIG. 4. Preoperative DSC and DCE acquisitions of an MM mass. A: Anatomical images with an overlaid ROI (traced with white lines) used for determining normalized CBV and kTrans. B: Color overlay to measure normalized CBVs in the interrogated ROI in the tumor area (indicated by \#1) and in control white matter (indicated by \#2); the normalized CBV is quite elevated, by approximately $17 \mathrm{ml}$. C: Color overlay to measure the kTrans in the interrogated ROI (indicated by \#1), yielding a kTrans of approximately $0.5 \mathrm{~min}^{-1}$. Figure is available in color online only.

2 tumor types. When microcystic and meningoepithelial variants were compared, a normalized CBV of $10.3 \mathrm{ml}$ gave a $100 \%$ PPV and a $71 \%$ NPV, with $100 \%$ specificity and $58 \%$ sensitivity.

A kTrans of greater than $0.88 \mathrm{~min}^{-1}$ yielded a PPV of $100 \%$ and a NPV of $93 \%$, with $100 \%$ specificity and $67 \%$ sensitivity for comparing MM with Grade IV astrocytomas. Similarly, in a comparison of MM with the meningoepithelial variant, a kTrans of greater than $0.88 \mathrm{~min}^{-1}$ gave a PPV of $100 \%$ and an NPV of $67 \%$, with $100 \%$ specificity and $67 \%$ sensitivity. Intraoperative blood loss tended to increase with increased normalized CBV $(\mathrm{R}=0.45)$, but this increase was not statistically significant $(p=0.085)$; the mean blood loss was $210 \mathrm{ml}$ (range 100-400 ml). Of note, none of the cases of MM had preoperative embolization.

\section{Discussion}

According to the WHO criteria, MMs are Grade I tu- mors. ${ }^{3}$ These tumors are morphologically distinct from the more common meningothelial tumors. ${ }^{11}$ The appearance of MMs on MRI sequences, in particular, the lack of a typical CSF cleft or dural tail in addition to the cystic nature of the tissue enhancement and surrounding vasogenic edema, may lead to preoperative planning that presumes the tumor to be an intrinsic high-grade glioma, rather than a benign extraaxial lesion. In addition, the high blood vessel density and increased permeability of these lesions suggest a potential for increased intraoperative blood loss. To date, anatomical imaging sequences have not enabled discrimination of MM from nonmicrocystic meningiomas or intrinsic primary brain tumors. In contrast, advanced imaging sequences such as perfusion-weighted imaging show promise to distinguish these tumor types before surgical exploration.

The normalized CBVs within the enhancing portion of MM were significantly higher than those observed in
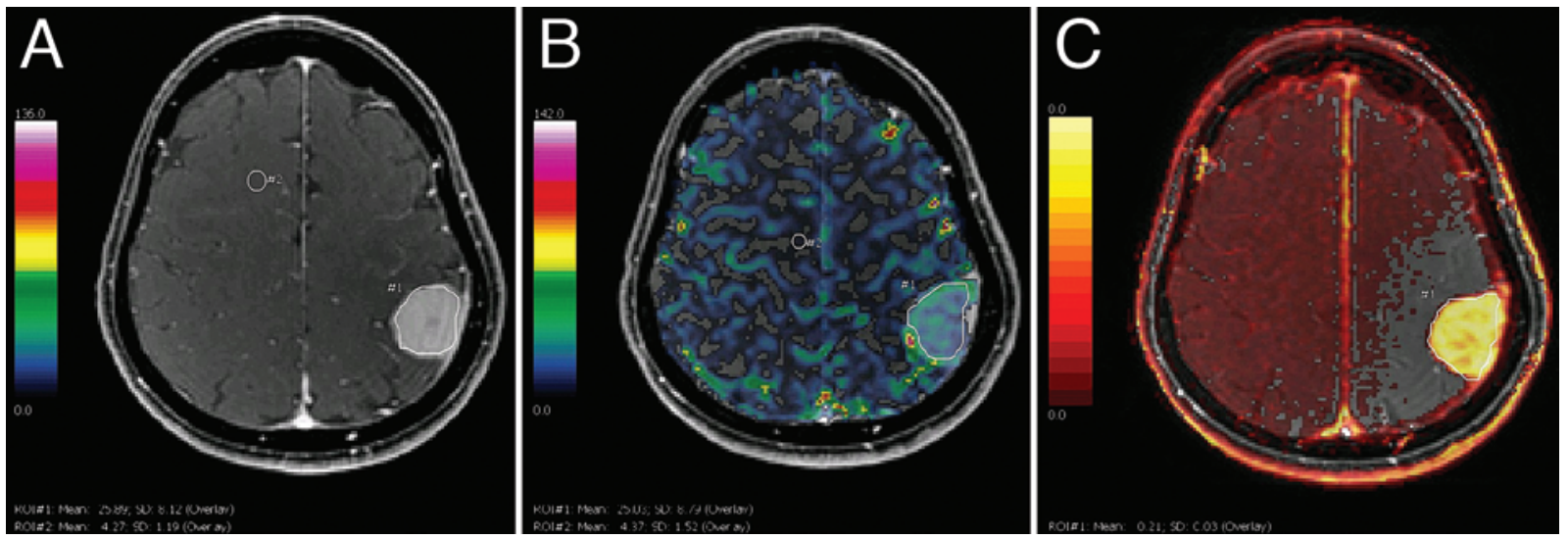

FIG. 5. Preoperative DSC and DCE acquisitions of a meningoepithelial meningioma. A: Anatomical images with an overlaid ROI (traced with white lines) used for determining normalized CBV and kTrans. B: Color overlay to measure normalized CBVs in the interrogated ROI in the tumor area (indicated by \#1) and in control white matter (indicated by \#2). The normalized CBV is elevated by approximately $6 \mathrm{ml}$. C: Color overlay to measure the kTrans in the interrogated ROI (indicated by \#1), yielding a kTrans of approximately $0.21 \mathrm{~min}^{-1}$. Figure is available in color online only. 

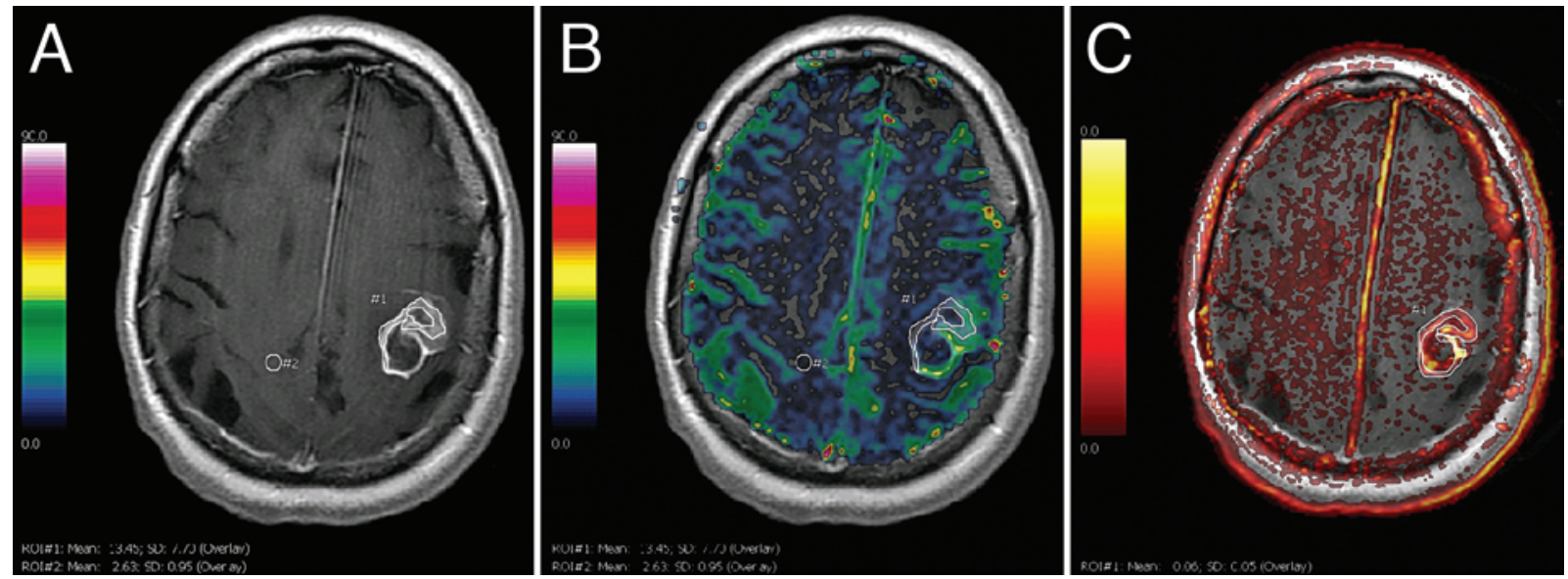

FIG. 6. Preoperative DSC and DCE acquisitions of a Grade IV astrocytoma A: Anatomical images with an overlaid ROI (traced with white lines) used for determining normalized CBV and kTrans. B: Color overlay to measure normalized CBVs in the interrogated ROI in the tumor area (indicated by \#1) and in control white matter (indicated by \#2). The normalized CBV is elevated by approximately $5 \mathrm{ml}$. C: Color overlay to measure the kTrans in the interrogated ROI (indicated by \#1), yielding a kTrans of approximately $0.06 \mathrm{~min}^{-1}$. Figure is available in color online only.

nonmicrocystic meningiomas or intrinsic primary brain tumors. In addition, DSC imaging observations within the T2 and FLAIR signal abnormalities surrounding an enhancing tumor can provide further support for a diagnosis of extrinsic as opposed to intrinsic tumors. The DSC measurements improve the diagnosis because although signals from extrinsic tumors tend to be comparable to those from normal white matter in meningiomas and metastatic tumors, T2 and FLAIR signals can be elevated in intrinsic primary brain tumors.

The histopathological substrate reflected in increased normalized CBVs appears to be microvascular density. ${ }^{2,7}$ To distinguish DSC imaging of tumors from that in stroke, we note the following essential considerations: leakage correction (mathematical or by contrast preload), arterial deconvolution (vs simpler mathematical techniques), choice of pharmacokinetic model, the time course and dosing used for preload, and consistent choice of an index tissue for normalization. These imaging parameters help to account for the inherent structural variability in tumor vasculature that distinguishes tumor blood flow from that observed in normal or ischemic tissue.

Jensen and Lee have reported on the utility of hypoxiainducible factor- $1 \alpha$, vascular endothelial growth factor, and cell proliferation indices such as MIB-1 to predict outcomes for patients with intracranial meningiomas and tumor recurrence. ${ }^{8}$ A correlation with tumor blood vessel density and a significant elevation in normalized CBV in MM do make intuitive sense when one considers the histopathological features that distinguish MM from nonmicrocystic meningiomas. Microcystic meningioma has a distinctly high density of blood vessels and vascular bundles, resulting in a higher net density of vasculature that increases the blood volume in MM tissue samples. This blood volume increase is then measured as a higher normalized CBV.

Use of perfusion-weighted imaging in the analysis of brain tumors has increased in relevance, as these imaging sequences help distinguish low-grade tumors from highgrade tumors, with normalized CBV being the most useful metric..$^{13}$ In both low- and high-grade primary tumors, nor- malized CBV appears to provide additional stratifying information by identifying more aggressive tumors. In addition, low-grade oligodendrogliomas may have an elevated normalized CBV without the aforementioned implications of aggressive behavior because of the increased capillary density in all grades of this histopathological subset.

Lui et al. used DSC and DCE imaging in a series of 20 patients to help identify and distinguish meningiomas from dural metastases. The authors reported that the normalized CBV parameter was not helpful in distinguishing tumor type, but that first-pass relative wash-in time was significantly $(p<0.05)$ lower for metastases than for meningiomas. ${ }^{14}$ This observation suggests the potential, as also noted in this study, for the continued use of contrast preload with subsequent acquisition of DCE and DSC sequences to distinguish intracranial tumor types.

Although a conclusive histopathological diagnosis cannot be made without obtaining tissue during the operation, these perfusion-weighted imaging measures, if obtained before surgery, provide the surgical team with additional information that may prepare them for intraoperative findings. Sequences obtained with DSC imaging may help identify more aggressive tumor subtypes within both lowand high-grade primary brain tumors, potentially influencing patient treatment, including the addition of adjunctive therapies. In addition, DSC imaging also has the potential for guiding treatment of patients with MM by predicting a histopathological diagnosis before surgery. Ideally, such approaches will provide information about whether extensive preparations for functional mapping are required (as might be more likely the case for invasive primary tumors) or whether blood should be ready on hand for transfusion.

As perfusion-weighted imaging becomes routine in many tumor-imaging protocols, an enhancing or cystic lesion with an unusually elevated normalized CBV should prompt clinicians and radiologists to consider the diagnosis of MM. Clinical personnel should factor such a diagnosis into their care paradigm as they prepare for surgical intervention. We conclude that the approach used in the present study may provide surgeons with additional infor- 
mation that could assist them in presurgical planning and better preparation for intraoperative diagnosis.

\section{Conclusions}

Identification of meningioma type based on preoperative imaging results can improve a surgeon's ability to meet the goals of patient care and ensure that the treatment paradigm and surgical goals are in line with the patient's best interests. Use of DSC and DCE sequences in the preoperative examinations of patients with meningioma provides surgeons with additional data and a better idea of what they might encounter during the operation. In addition, the findings obtained with these imaging techniques could help predict a patient's postoperative outcomes. In planning surgical care, the patient care team should consider an enhancing cystic lesion with elevated normalized CBV of greater than $10.3 \mathrm{ml}$ or increased kTrans of greater than $0.88 \mathrm{~min}^{-1}$ to indicate $\mathrm{MM}$ rather than traditional Grade I nonmicrocystic meningioma or intrinsic high-grade glioma.

\section{References}

1. Awasthi R, Rathore RK, Soni P, Sahoo P, Awasthi A, Husain $\mathrm{N}$, et al: Discriminant analysis to classify glioma grading using dynamic contrast-enhanced MRI and immunohistochemical markers. Neuroradiology 54:205-213, 2012

2. Barajas RF Jr, Phillips JJ, Parvataneni R, Molinaro A, Essock-Burns E, Bourne G, et al: Regional variation in histopathologic features of tumor specimens from treatment-naive glioblastoma correlates with anatomic and physiologic MR imaging. Neuro Oncol 14:942-954, 2012

3. Burger PC: Revising the World Health Organization (WHO) Blue Book-'Histological typing of tumours of the central nervous system.' J Neurooncol 24:3-7, 1995

4. Chen CJ, Tseng YC, Hsu HL, Jung SM: Microcystic meningioma: importance of obvious hypointensity on T1-weighted magnetic resonance images. J Comput Assist Tomogr 32:130-134, 2008

5. Cuccurullo L, Parlato C, Luongo M, Accardo M: Ultrastructural profile of microcystic meningioma. Pathologica 101:115-118, 2009

6. Haacke EM, Li M, Juvvigunta F: Tissue similarity maps (TSMs): a new means of mapping vascular behavior and calculating relative blood volume in perfusion weighted imaging. Magn Reson Imaging 31:481-489, 2013

7. Hu LS, Eschbacher JM, Heiserman JE, Dueck AC, Shapiro WR, Liu S, et al: Reevaluating the imaging definition of tumor progression: perfusion MRI quantifies recurrent glioblastoma tumor fraction, pseudoprogression, and radiation necrosis to predict survival. Neuro Oncol 14:919-930, 2012

8. Jensen R, Lee J: Predicting outcomes of patients with intracranial meningiomas using molecular markers of hypoxia, vascularity, and proliferation. Neurosurgery 71:146-156, 2012

9. Jensen-Kondering U, Henker C, Dörner L, Hugo HH, Jansen O: Differentiation of primary central nervous system lymphomas from high grade astrocytomas by qualitative analysis of the signal intensity curves derived from dynamic susceptibility-contrast magnetic resonance imaging. Neurol Res 34:984-988, 2012

10. Kim J, Leira EC, Callison RC, Ludwig B, Moritani T, Magnotta VA, et al: Toward fully automated processing of dynamic susceptibility contrast perfusion MRI for acute ischemic cerebral stroke. Comput Methods Programs Biomed 98:204-213, 2010

11. Kuchna I, Matyja E, Wierzba-Bobrowicz T, Mazurowski W:
Microcystic meningioma--a rarely occurring morphological variant of meningioma. Folia Neuropathol 32:259-263, 1994

12. Larsson HB, Courivaud F, Rostrup E, Hansen AE: Measurement of brain perfusion, blood volume, and blood-brain barrier permeability, using dynamic contrast-enhanced $\mathrm{T}(1)$ weighted MRI at 3 tesla. Magn Reson Med 62:1270-1281, 2009

13. Law M, Young RJ, Babb JS, Peccerelli N, Chheang S, Gruber ML, et al: Gliomas: predicting time to progression or survival with cerebral blood volume measurements at dynamic susceptibility-weighted contrast-enhanced perfusion MR imaging. Radiology 247:490-498, 2008

14. Lui YW, Malhotra A, Farinhas JM, Dasari SB, Weidenheim K, Freeman K, et al: Dynamic perfusion MRI characteristics of dural metastases and meningiomas: a pilot study characterizing the first-pass wash-in phase beyond relative cerebral blood volume. AJR Am J Roentgenol 196:886-890, 2011

15. Ozsunar Y, Mullins ME, Kwong K, Hochberg FH, Ament C, Schaefer PW, et al: Glioma recurrence versus radiation necrosis? A pilot comparison of arterial spin-labeled, dynamic susceptibility contrast enhanced MRI, and FDG-PET imaging. Acad Radiol 17:282-290, 2010

16. Pinto PS, Huisman TA, Ahn E, Jordan LC, Burger P, Cohen $\mathrm{KJ}$, et al: Magnetic resonance imaging features of meningiomas in children and young adults: a retrospective analysis. J Neuroradiol 39:218-226, 2012

17. Prabhakaran V, Raman SP, Grunwald MR, Mahadevia A, Hussain N, Lu H, et al: Neural substrates of word generation during stroke recovery: the influence of cortical hypoperfusion. Behav Neurol 18:45-52, 2007

18. Rishi A, Black KS, Woldenberg RW, Overby CM, Eisenberg MB, Li JY: Microcystic meningioma presenting as a cystic lesion with an enhancing mural nodule in elderly women: report of three cases. Brain Tumor Pathol 28:335-339, 2011

19. Valotassiou V, Leondi A, Angelidis G, Psimadas D, Georgoulias P: SPECT and PET imaging of meningiomas. ScientificWorldJournal 2012:412580, 2012

20. Wang DJ, Alger JR, Qiao JX, Hao Q, Hou S, Fiaz R, et al: The value of arterial spin-labeled perfusion imaging in acute ischemic stroke: comparison with dynamic susceptibility contrast-enhanced MRI. Stroke 43:1018-1024, 2012

21. Xyda A, Haberland U, Klotz E, Bock HC, Jung K, Knauth M, et al: Brain volume perfusion CT performed with 128-detector row CT system in patients with cerebral gliomas: a feasibility study. Eur Radiol 21:1811-1819, 2011

\section{Disclosures}

The authors report no conflict of interest concerning the materials or methods used in this study or the findings specified in this paper.

\section{Author Contributions}

Conception and design: Hussain, Keogh, Fouke. Acquisition of data: Hussain, Keogh, Rostad, Aguedan, Good. Analysis and interpretation of data: Hussain, Keogh, McCullough, Rostad. Drafting the article: Hussain, Keogh. Critically revising the article: Hussain, Moisi, Keogh, Rostad, Fouke. Reviewed submitted version of manuscript: Hussain, Moisi, Keogh. Statistical analysis: Hussain, McCullough. Administrative/technical/material support: Hussain, Newell, Gwinn, Mayberg. Study supervision: Hussain, Keogh, Foltz.

\section{Correspondence}

Namath S. Hussain, Swedish Neuroscience Institute, Swedish Medical Center, 550 17th Ave., Seattle, WA 98122. email: namath.hussain@gmail.com. 